\title{
Percepção de estudantes de Fonoaudiologia sobre a Supervisão Clínica
}

\author{
Perception of students \\ on the Clinical Supervision of Speech, \\ Language and Hearing Sciences
}

\section{Percepción de estudiantes de Fonoaudiología sobre la Supervisión Clínica}

\author{
Patrícia Pupin Mandrá* \\ Rita Cristina Sadako Kuroishi* \\ Nathaly Anne de Souza Gomes* \\ Matheus Francoy Alpes*
}

\section{Resumo}

Investigou-se a percepção de estagiários de um Curso de Fonoaudiologia sobre a supervisão clínica. Um instrumento dirigido com escala Likert (0-5) foi preenchido por 44 estudantes do $6^{\circ}$ e $8^{\circ}$ período e analisados por estatística não paramétrica. Houve maior percentual de respostas para "concordo parcialmente" (CP) ou "concordo totalmente" (CT) para a Percepção sobre a Supervisão da Prática Profissionalizante (PSPP), Percepção sobre a Função do Supervisor (PFS) e Percepção do Papel do Estagiário (PPE). No PSPP, embora predominassem respostas CT, 100\% dos participantes não opinaram sobre "A supervisão fortalece o embasamento teórico e o raciocínio clínico". No PFS, na questão identificação e discussão dos sentimentos e reações do estagiário, $43,18 \%$ para $\mathrm{CP}$, seguido por $20,45 \%$ para DP. No PPE, 34,09\% discordaram parcialmente que a expectativa, tensão e ansiedade influenciam negativamente o aprendizado, seguido de $25,00 \%$ para CT. Evidenciou-se importância do supervisor, estagiário e supervisão clínica, como modelo de ensino-aprendizagem para aquisição e aprimoramento de habilidades e competências necessárias para a prática profissional.

Palavras-chave: Estágio Clínico; Ensino; Aprendizagem; Fonoaudiologia

*Faculdade de Medicina de Ribeirão Preto - FMRP/USP, Ribeirão Preto, SP, Brasil.

Contribuição dos Autores:

PPM- orientadora da pesquisa definindo os objetivos e método, acompanhando as etapas de coleta e análise de dados, bem como a elaboração e revisão final do artigo; NASG- responsável pela coleta e análise de dados; RCSK e MFA- auxilio no processo de análise de dados e elaboração do artigo.

Email para correspondência: Matheus Francoy Alpes matheus.alpes@usp.br Recebido: 21/12/2018

Aprovado: 20/05/2019 


\section{Abstract}

The perception of trainees in Speech, Language and Hearing Sciences about clinical supervision was investigated. Data were collected using a Likert scale instrument (0-5), completed by 44 students from the 6 th and 8 th periods, analyzed by non-parametric statistics. There was a higher percentage of responses to "Partially Agree" (PA) or "Totally Agree" (TA) for Perception on Supervision of Professional Practice (PSPP), Perception on Supervisor Role (PFR) and Trainee Perception of Role (TPR). In the PSPP, although TA responses predominated, $100 \%$ of the participants did not comment on "Supervision strengthens the theoretical basis and clinical reasoning". In the PFR, in the question identification and discussion of the feelings and reactions of the trainee, $43.18 \%$ for PA, followed by $20.45 \%$ for PD. In TPR, $34.09 \%$ partially disagreed that expectation, tension and anxiety negatively influence learning, followed by $25.00 \%$ for TA. The importance of supervisor, trainee and clinical supervision was demonstrated as a teaching-learning model to acquire and improve the skills and competencies required for professional practice.

Keywords: Clinical Clerkship; Teaching; Learning; Speech, Language and Hearing Sciences

\section{Resumen}

Se investigó la percepción de pasantes de un Curso de Fonoaudiología sobre la supervisión clínica. Los datos recogidos con un instrumento dirigido con escala Likert (0-5), llenado por 44 estudiantes del $6^{\circ}$ y $8^{\circ}$ período, analizados por estadística no paramétrica. Hubo un mayor porcentaje de respuestas a "Parcialmente de acuerdo" (PA) o "Totalmente de acuerdo" (TA) para Percepción de supervisión de práctica profesional (PSPP), Percepción de rol de supervisor (PRS) y Percepción de rol de aprendiz (PRA). En el PSPP, aunque predominaban respuestas TA, el 100\% de los participantes no opinaron sobre "La supervisión fortalece el fundamento teórico y el raciocinio clínico". En el PRS, en la cuestión identificación y discusión de los sentimientos y reacciones del pasante, el 43,18\% para PA, seguido por el 20,45\% para DP. En el PRA, el 34,09\% discrepó parcialmente que la expectativa, tensión y ansiedad influencian negativamente el aprendizaje, seguido del $25,00 \%$ para TA. Se evidenció la importancia del supervisor, pasante y supervisión clínica, como modelo de enseñanza-aprendizaje para adquisición y perfeccionamiento de habilidades y competencias necesarias para la práctica profesional.

Palabras claves: Estágio Clínico; Ensino; Aprendizagem; Fonoaudiologia

\section{Introdução}

O ensino superior em saúde é fundamental na sociedade, pois os egressos dos cursos ofertados na área serão os profissionais que prestarão assistência à população ${ }^{1}$. A prática profissionalizante durante $\mathrm{a}$ graduação constitui o primeiro momento de inserção do futuro profissional no campo de atuação². Seus principais objetivos consistem em fortalecer o embasamento teórico, estabelecer conduta ética e garantir a competência clínica ${ }^{3}$. Busca-se desenvolver habilidades atitudinais e postura profissional por meio de relato dos atendimentos clínicos, debate sobre os projetos referentes ao planejamento das atividades, troca de experiências com o coletivo de formação, aquisição de conhecimento sobre a ética e a técnica, além de abordar as dificuldades e questões relacionadas à vivência clínica do estagiário ${ }^{4}$.
Ao entender a supervisão de estágio como promotora de crescimento, ressalta-se sua peculiaridade e importância no âmbito acadêmico ${ }^{4}$, principalmente quando se exige competência, responsabilidade, trabalho em equipe e desenvolvimento colaborativo de saberes, entendendo-se a formação destes profissionais como um processo contínuo de apropriação pessoal do saber em contextos interativos ${ }^{5}$.

A transição entre o papel de aluno para o de estagiário não é uma tarefa fácil de ser vivenciada e o processo de avaliação do seu desempenho pelo docente pode acarretar angústias e conflitos na relação entre os participantes do grupo ou com o próprio supervisor. Assim, o manejo de diferentes estratégias para lidar com as dificuldades e desenvolver as potencialidades do aprendiz tem papel primordial ${ }^{6}$. Durante o processo de ensino-apren- 
dizagem, o supervisor deve conceber o estudante como agente da construção do seu próprio conhecimento, e assumir que com o diálogo entre supervisor e os demais estagiários haverá a possibilidade de compartilhar as experiências e enfatizar o ato de reflexão ${ }^{7}$ e construção de raciocínio.

Enquanto atividade acadêmica, a supervisão sempre estará sujeita a mudanças de paradigmas teóricos, decorrentes dos avanços científicos e das necessidades sociais ${ }^{7}$. É necessário capacitar os supervisores/preceptores para garantir maior qualidade no estabelecimento de critérios para avaliar o desempenho, para realizar as observações da atuação dos estudantes, para realizar a prática de provimento de informações (feedback contínuo) e acompanhar o desenvolvimento das competências esperadas $^{8}$ durante o processo.

$\mathrm{Na}$ área de psicologia tem-se o reconhecimento na literatura da falta de modelos e sistematização da supervisão clínica, permanecendo uma diversidade de maneiras e métodos relacionados à formação pessoal do supervisor, porém sem a avaliação do processo ou resultado?.

O estudo criterioso e sistemático do estágio obrigatório na área de Fonoaudiologia é considerado um dos principais caminhos para a melhor compreensão da formação deste profissional e há escassez de estudos sobre a supervisão nesta área ${ }^{10}$. Ao investigar o perfil do supervisor de estágio na área de Fonoaudiologia e como ocorre a sua prática durante o acompanhamento dos estagiários, evidenciou-se a necessidade de formação específica voltada para a docência, assim como do redirecionamento da formação para a Atenção Básica e Interdisciplinaridade ${ }^{11}$.

As estratégias de ensino-aprendizagem e o processo de avaliação de estudantes da área da saúde vêm sendo discutidos mundialmente, principalmente para a formação médica. Para o aprendizado de adultos, deve-se considerar como uma variável, o ambiente educacional, o que incluiria o cenário de prática e o supervisor. Este ambiente influencia o desenvolvimento dos atributos esperados para um profissional da saúde relacionados a: conhecimento, habilidades e atitude. Este conjunto de habilidades de diversas naturezas devem ser exploradas pelo supervisor/preceptor durante o ensino em cenário atuação (clínico) real. Neste ambiente, podem-se utilizar diferentes estratégias que irão favorecer o processo de ensino-aprendizagem nos diferentes locais de estágio (hospital, centro de reabilitação, unidade básica de saúde, estratégia de saúde da família, domicílios e equipamentos sociais). Cabe a quem ensina reconhecer conhecimento prévio de quem aprende e o encorajamento do aprendizado com pares durante a supervisão.

A prática profissionalizante supervisionada é considerada uma das mais eficientes para o desenvolvimento das competências clínicas. Sendo assim, o objetivo do presente estudo foi investigar a percepção dos estagiários de um curso de Fonoaudiologia sobre a supervisão clínica, como modelo do processo ensino-aprendizagem.

\section{Método}

O estudo foi aprovado pelo Comitê de Ética em Pesquisa do Hospital das Clínicas de Ribeirão Preto da Faculdade de Medicina de Ribeirão Preto (HCFMRP-USP), mediante o parecer consubstanciado n³929/2010. Os participantes assinaram o Termo de Consentimento Livre e Esclarecido (TCLE).

Foram selecionados todos os estudantes que já haviam cursado as disciplinas de prática profissionalizante (clínicas) do $3^{\circ}$ e $4^{\circ}$ anos do curso. Participaram 44 estudantes, sendo 26 matriculados no $6^{\circ}$ período $(59 \%)$ e 18 matriculados no $8^{\circ}$ período (41\%). A média de idade foi de 22 anos e 100\% eram do sexo feminino.

O instrumento elaborado para a coleta era composto de guia instrucional e 26 questões distribuídas em três blocos de conteúdo: a) Percepção sobre a Supervisão da Prática Profissionalizante (PSPP: 10 questões), b) Percepção sobre a Função do Supervisor (PFS: 11 questões) e, c) Percepção do Papel do Estagiário (PPE: cinco questões). Foi estabelecida uma escala de resposta psicométrica do tipo Likert (1-3) para que o participante assinalasse uma das seguintes opções: (1) Discordo (D), (2) Não posso opinar (NPO), (3) Concordo (C). A coleta foi realizada em sala do bloco didático da Instituição. Durante o procedimento o examinador não fez interrupção ou qualquer observação que pudesse interferir nas respostas.

Os questionários foram lidos e separados por período de graduação e as respostas foram organizadas em planilhas do Excel® para análise descritiva através do cálculo de frequências absolutas e relativas. 


\section{Resultados}

Obteve-se maior percentual de concordância para o conteúdo pesquisado nos três blocos investigados.

Os participantes $(100 \%)$ afirmaram não poder opinar sobre a afirmação que " $A$ supervisão fortalece o embasamento teórico e o raciocínio clínico" (Tabela 1).
Na Tabela 2, observa-se que houve discordância sobre algumas funções atribuídas ao supervisor: $31,81 \%$ para a identificação e discussão dos sentimentos e reações do estagiário (B10) e $25 \%$ para o feedback periódico (B6). Porém, 68,19\% concordaram que o supervisor deve perceber e considerar diferenças individuais (B11).

Tabela 1. Percepção sobre a Supervisão da Prática Profissionalizante (PSPP)

\begin{tabular}{|c|c|c|c|c|c|c|}
\hline \multirow{2}{*}{ Afirmações } & \multicolumn{2}{|c|}{ D } & \multicolumn{2}{|c|}{ NPO } & \multicolumn{2}{|c|}{$\mathbf{C}$} \\
\hline & $\mathbf{n}$ & $\%$ & $\mathbf{n}$ & $\%$ & $\mathbf{n}$ & $\%$ \\
\hline Fortalece o embasamento teórico (A1) & 0 & 0 & 44 & 100 & 0 & 0 \\
\hline Desenvolve postura e conduta profissional (A2) & 0 & 0 & 3 & 6,82 & 40 & 90,91 \\
\hline $\begin{array}{l}\text { Desenvolve o relacionamento e comunicação interpessoal } \\
\text { em grupos (A3) }\end{array}$ & 2 & 4,55 & 1 & 2,27 & 41 & 93,19 \\
\hline $\begin{array}{l}\text { Desenvolve a iniciativa e autonomia para a resolução dos } \\
\text { problemas clínicos (A4) }\end{array}$ & 0 & 0 & 4 & 9,09 & 40 & 90,91 \\
\hline Colabora para o planejamento e execução das ações (A5) & 0 & 0 & 2 & 4,55 & 42 & 95,45 \\
\hline $\begin{array}{l}\text { Desenvolve a percepção de suas próprias dificuldades por } \\
\text { meio da autocrítica e autoconhecimento (A6) }\end{array}$ & 0 & 0 & 2 & 4,55 & 42 & 95,45 \\
\hline $\begin{array}{l}\text { Desenvolve a reflexão crítica sobre condutas, intervenções } \\
\text { e evolução dos casos (A7) }\end{array}$ & 1 & 2,27 & 2 & 4,55 & 41 & 93,18 \\
\hline $\begin{array}{l}\text { Reflexão crítica sobre o manuseio de equipamentos e } \\
\text { exceção de técnicas (A8) }\end{array}$ & 0 & 0 & 1 & 2,27 & 42 & 95,46 \\
\hline $\begin{array}{l}\text { Discussão de conteúdos de Plano Diário e relatórios para } \\
\text { modificar conduta (A9) }\end{array}$ & 1 & 2,27 & 4 & 9,09 & 39 & 88,63 \\
\hline $\begin{array}{l}\text { Apresentação e discussão de todos os casos como fonte de } \\
\text { aprendizado (A10) }\end{array}$ & 1 & 2,27 & 1 & 2,27 & 42 & 95,45 \\
\hline
\end{tabular}

Legenda: D (Discordo), NPO (Não posso opinar) e C (Concordo)

Tabela 2. Percepção sobre a Função do Supervisor (PFS)

\begin{tabular}{|c|c|c|c|c|c|c|}
\hline \multirow{2}{*}{ Afirmações } & \multicolumn{2}{|c|}{ D } & \multicolumn{2}{|c|}{ NPO } & \multicolumn{2}{|c|}{ C } \\
\hline & $\mathbf{n}$ & $\%$ & $\mathbf{n}$ & $\%$ & $\mathbf{n}$ & $\%$ \\
\hline $\begin{array}{l}\text { Organizar pedagogicamente a supervisão de forma } \\
\text { dinâmica (B1) }\end{array}$ & 1 & 2,27 & 0 & 0 & 43 & 97,73 \\
\hline $\begin{array}{l}\text { Apresentar e discutir os critérios de avaliação de } \\
\text { desempenho (B2) }\end{array}$ & 3 & 6,82 & 2 & 4,55 & 39 & 88,63 \\
\hline $\begin{array}{l}\text { Estimular o desenvolvimento de habilidades e competências } \\
\text { do estagiário (B3) }\end{array}$ & 3 & 6,82 & 3 & 6,82 & 38 & 86,36 \\
\hline $\begin{array}{l}\text { Articular as ações de ensino em assistência aos resultados } \\
\text { de pesquisas atuais da área (B4) }\end{array}$ & 4 & 9,09 & 5 & 11,36 & 35 & 79,55 \\
\hline $\begin{array}{l}\text { Incentivar o estudo teórico do caso e conduzir ao raciocínio } \\
\text { clínico (B5) }\end{array}$ & 5 & 11,36 & 2 & 4,55 & 37 & 84,09 \\
\hline $\begin{array}{l}\text { Informar e discutir periodicamente sobre a necessidade de } \\
\text { mudanças para melhorar o desempenho (B6) }\end{array}$ & 11 & 25 & 3 & 6,82 & 30 & 68,19 \\
\hline $\begin{array}{l}\text { Promover reflexão sobre o mercado de trabalho, inserção } \\
\text { na carreira e as diversas faces da atuação profissional (B7) }\end{array}$ & 5 & 11,36 & 5 & 11,36 & 34 & 77,27 \\
\hline $\begin{array}{l}\text { Anotar em Plano Diário a observação para facilitar o } \\
\text { planejamento das ações futuras (B8) }\end{array}$ & 5 & 11,36 & 2 & 4,55 & 38 & 86,36 \\
\hline Incentivar a apresentação e discussão dos casos clínicos (B9) & 4 & 9,09 & 1 & 2,27 & 39 & 88,63 \\
\hline $\begin{array}{l}\text { Identificar e discutir sentimentos e reações do estagiário } \\
\text { (B10) }\end{array}$ & 14 & 31,81 & 4 & 9,09 & 26 & 59,09 \\
\hline $\begin{array}{l}\text { Perceber e considerar as diferenças individuais dos } \\
\text { membros do grupo (B11) }\end{array}$ & 10 & 22,73 & 4 & 9,09 & 30 & 68,19 \\
\hline
\end{tabular}

Legenda: D (Discordo), NPO (Não posso opinar) e C (Concordo) 
Quanto à percepção do papel de estagiário, $50,77 \%$ discordaram que a expectativa, tensão e ansiedade influenciam negativamente o apren- dizado (C1). E apontaram para o papel ativo do estudante no planejamento e embasamento teórico das ações (C4 E C5).

Tabela 3. Percepções sobre Percepção do Papel do Estagiário (PPE)

\begin{tabular}{|c|c|c|c|c|c|c|}
\hline \multirow{2}{*}{ Afirmações } & \multicolumn{2}{|c|}{ D } & \multicolumn{2}{|c|}{ NPO } & \multicolumn{2}{|c|}{ C } \\
\hline & $\mathbf{n}$ & $\%$ & $\mathbf{n}$ & $\%$ & $\mathbf{n}$ & $\%$ \\
\hline $\begin{array}{l}\text { Expectativa negativa, tensão e ansiedade interferência } \\
\text { durante primeiro atendimento ( } 1 \text { ) }\end{array}$ & 21 & 50,77 & 3 & 6,82 & 20 & 45,45 \\
\hline $\begin{array}{l}\text { É responsável por planejar e aplicar técnica durante } \\
\text { atendimento (C2) }\end{array}$ & 5 & 11,36 & 2 & 4,55 & 37 & 84,09 \\
\hline $\begin{array}{l}\text { Deve compreender e obedecer ao regulamento dos } \\
\text { diferentes cenários de prática (C3) }\end{array}$ & 0 & 0 & 5 & 11,36 & 39 & 88,63 \\
\hline $\begin{array}{l}\text { Deve participar ativamente no desenvolvimento do } \\
\text { raciocínio clínico para a discussão de caso (C4) }\end{array}$ & 0 & 0 & 2 & 4,55 & 42 & 95,45 \\
\hline $\begin{array}{l}\text { Deve revisar a literatura para embasar o planejamento } \\
\text { das condutas e discussão dos casos (C5) }\end{array}$ & 1 & 2,27 & 1 & 2,27 & 42 & 95,46 \\
\hline
\end{tabular}

Legenda: D (Discordo), NPO (Não posso opinar) e C (Concordo)

\section{Discussão}

A supervisão é uma estratégia usada para o desenvolvimento de habilidades e competências ${ }^{3,7} \mathrm{em}$ ambiente real (cenário de prática profissionalizante) de ensino e aprendizado. Considera-se que este modelo favorece o desenvolvimento e construção de uma autonomia intelectual e profissional ${ }^{2}$ sendo, portanto, um momento crucial para a transição entre a identidade de estagiário para a de profissional ${ }^{11}$.

A experiência positiva relacionada ao estágio já foi constatada por estudos com alunos de cursos de Enfermagem. De acordo com os resultados publicados, a supervisão contribuiu para que estudantes de enfermagem pudessem perceber a relação entre o conhecimento cognitivo (saber) adquirido previamente e a atuação em serviço, o que promoveu o crescimento pessoal, a aquisição de autoconfiança e foi fundamental para capacitá-los para a atuação como futuros profissionais no mercado de traba1 ho ${ }^{12-13}$. Observou-se que os estudantes de fonoaudiologia participantes desta pesquisa não opinaram sobre a relação entre a supervisão e o fortalecimento da teoria. Na instituição em questão, a atividade em cenário de prática profissionalizante é dividida em três momentos: a) o planejamento prévio de metas e estratégias de ação (redação de plano diário), b) a realização dos procedimentos (atendimento) com a observação do preceptor e, c) a supervisão em pequeno grupo com a estratégia de long case e feedback imediato. Com este modelo, pretende-se desenvolver habilidades de comunicação/interação em equipe, objetividade, tomada de decisão, solução de problemas e raciocínio clínico com base em princípios teóricos. A maioria $(90,91 \%)$ dos estudantes concordou que a estratégia de supervisão adotada era importante para o desenvolvimento da postura e conduta profissional ética (A2), bem como das habilidades que garantiam a competência clínica (A3;A4;A5;A6;A7;A8;A9;A10). Apesar de não haver evidência precisa do que a torna a supervisão efetiva, autores referiram que a diversidade de docente/preceptores qualificados aumentaria a objetividade dos resultados finais ${ }^{14}$.

Vale ressaltar que as Diretrizes Curriculares Nacionais sugerem a inserção de estudantes da área da saúde no âmbito do SUS e que os participantes desta pesquisa já tinham realizado prática profissionalizante nos seguintes cenários: atenção básica (estratégia de saúde da família), centro integrado de reabilitação (média complexidade), ambulatórios e enfermarias de hospital (média e alta complexidade), ou seja, em diferentes níveis de complexidade. Esta característica torna importante o preparo do ambiente educacional no serviço de saúde, o que envolveria a estrutura física (sala para atendimento e supervisão), equipamentos e instrumentos e os recursos humanos, neste caso o preceptor (profissional local). Cabe aos docentes supervisores, articular e/ou adequar o conteúdo das disciplinas ao ambiente educacional para favorecer 
o desenvolvimento de competências procedimentais, atitudinais e afetivas.

Para que haja integração entre as atividades de prestação de serviço à comunidade e o processo de ensino-aprendizagem de qualidade, é fundamental que os preceptores/supervisores conheçam o Projeto Político Pedagógico do Curso, sua matriz curricular, bem como os objetivos de aprendizagem (conteúdo/habilidade), estratégia de ensino-aprendizagem e método de avaliação de desempenho do conjunto de disciplinas de prática profissionalizante $^{15}$.

A falta de experiência em atuar clinicamente levará o estagiário a buscar o apoio do supervisor (docente) e/ou preceptor local ${ }^{16}$. E o docente/supervisor deverá direcionar o olhar do aluno para que o vínculo indissociável entre a prática clínica e o conhecimento teórico seja estabelecido ${ }^{1}$, e para que todos os problemas sejam discutidos e teorizados para o planejamento de soluções ${ }^{17}$.

Os supervisores/preceptores locais devem ser incentivados a basear sua prática em evidências clínicas comprovadas cientificamente, mantendo-se constantemente atualizados, já que são modelos de aprendizado. Ser supervisor/preceptor exige múltiplas competências, que ultrapassam as habilidades procedimentais (seleção e execução de técnicas), incluindo o monitoramento constante de suas atitudes profissionais relacionadas à postura, responsabilidade, comportamento ético e acolhimento humanizado. Constatou-se que $84,09 \%$ afirmaram que foram incentivados a estudar (teorizar) o caso e realizar o raciocínio clínico (B5), confirmando a importância do supervisor para criar um ambiente que favoreça o aprendizado significativo, sendo também importante que ele reconheça a competência cognitiva (saber) prévia do estudante e o auxilie a buscar novas possibilidades para a leitura científica que sustente sua atuação ${ }^{18}$. Durante as práticas, os estudantes do curso estudado produzem relatórios clínicos e de hipótese diagnóstica e ambos devem ser pautados na literatura científica. As devolutivas (orais e escritas) sobre este material são consideradas um momento de reflexão e aprendizado.

Os participantes concordaram que os estágios promoveram a iniciativa e autonomia para o estudo clínico de caso e resolução de problemas clínicos (A4, 93,19\%). E confirmaram a importância da pró-atividade e responsabilidade deles mesmos com seu aprendizado (C4 e C5, 95,45\%). Durante a supervisão, os estudantes são incentivados a apresentar e problematizar seus casos (estratégia long case), e assim, ideias são discutidas e corroboradas com a teoria, o que leva o grupo de estudantes juntamente com o supervisor a discutir e escolher a melhor opção para a condução do caso. $\mathrm{O}$ aprendizado colaborativo acontece com a troca de experiência no grupo de supervisão, que na Instituição é composto por 8 estudantes, o docente coordenador do estágio, o preceptor local e monitor da disciplina (estudante do último ano do curso).

A estratégia de discussão em pequeno grupo é predominantemente centrada no estudante, o que favorece o desenvolvimento de habilidades sociais e de comunicação (ouvir, debater, argumentar, entre outras). E, juntamente com a relação com as equipes do serviço de saúde são extremamente importantes para desenvolver as habilidades do relacionamento interpessoal e respeito (A2). Conviver com as diferentes perspectivas e pessoas prepara o estudante para o trabalho em equipe ${ }^{18} \mathrm{e}$ para diferentes cenários de ocupação profissional. Uma pesquisa anterior enfatizou a importância das interações sociais e cooperação mútua entre os integrantes do grupo de estágio, afirmando que as trocas de conhecimentos e de possibilidades de ação favorecem o processo de mudança, confirmando que o estágio supervisionado em serviço de saúde prepara o estudante para o enfrentamento da realidade do mercado de trabalho ${ }^{19}$. Os estudantes pesquisados consideraram que o incentivo a apresentação e discussão dos casos clínicos $(88,63 \%)$ (B9) e incentivo à reflexão sobre o mercado de trabalho e as diversas faces da atuação profissional $(77,27 \%)$ (B7) são responsabilidade do supervisor.

A experiência possibilita ao estagiário adquirir conhecimento técnico-prático, proporcionando uma reflexão crítica que o leva à autoconfiança. O pensamento crítico-reflexivo o tornará um profissional consciente de seu papel na sociedade, possibilitando um atendimento mais humanizado e diferenciado, implicando em uma melhora na qualidade do serviço prestado ao usuário ${ }^{19}$.

O manejo realizado pelo supervisor para lidar com as dificuldades e potencialidades dos estagiários é importante, cabendo ao mesmo, decodificar e transformar tais sentimentos, a fim de promover o entendimento dos processos psíquicos que permeiam as relações em grupo, porém sem inferir sobre questões pessoais e/ou expô-lo ao grupo. Constatou-se que 59,09\% consideraram 
importante o supervisor identificar e discutir os sentimentos e reações do estagiário (B10); 50,77\% destes participantes discordaram quanto à interferência negativa de expectativa, tensão e ansiedade no primeiro atendimento $(\mathrm{C} 1)$. A resposta obtida na questão B10 demonstra que o supervisor deve mediar as relações e os sentimentos envolvidos nas competências múltiplas do supervisor.

Estagiários de um curso de Fonoaudiologia em cenário hospitalar referiram-se diretamente ao impacto emocional inicial e citaram sentimentos negativos, tais como ansiedade, frustração, insegurança, sensação de incapacidade, angústia, tristeza e medo ${ }^{20}$, durante a prática profissionalizante.

Diferentes cenários de prática em serviço de saúde exigem atitude, habilidade e competência diversificadas e a empatia do estudante com o perfil de atendimento realizado. Os participantes da pesquisa já haviam realizado atividade em atenção básica, centro de reabilitação de média complexidade e ambulatórios e enfermarias de alta complexidade em hospital.

O posicionamento do supervisor durante o feedback e a adoção de técnica de comunicação não violenta podem ser responsáveis pela promoção de um ambiente educativo favorável ou desfavorável ao acolhimento das necessidade do estagiário e da diversidade ${ }^{20}(\mathrm{~B} 9, \mathrm{~B} 10, \mathrm{~B} 11)$. De acordo com $68,18 \%$ dos participantes é função do supervisor perceber e considerar as diferenças individuais dentro do grupo (B11).

A interação permanente com o supervisionando, partilhando uma prática reguladora da ação, é uma prática reflexiva que contribui para o desenvolvimento de ambos os atores envolvidos ${ }^{6}$, sendo que o supervisor deve ter competências sociais para saber conviver com o outro, estabelecendo uma relação respeitosa e dialógica ${ }^{20}$.

Segundo a opinião dos participantes $(97,73 \%)$, as atividades de estágio devem ser organizadas com princípios pedagógicos e de forma dinâmica (B1), e os critérios de avaliação de desempenho devem ser apresentados e discutidos (88,63\%) (B2). Como estratégias de ensino aprendizagem concordaram que é importante que o supervisor articule as ações de ensino em assistência aos resultados de pesquisas atuais da área (86,36\%) (B4), que estimule o desenvolvimento de habilidades e competências profissionais $(88,63)(\mathrm{B} 3)$, realizando devolutivas periódicas apontando para a necessidade e o caminho para a mudança de atitude $(68,19 \%)$ (B6).
Com relação à avaliação do desempenho em atividades de estágio, notou-se que a maioria dos participantes concordou que é responsabilidade do supervisor apresentar e discutir os critérios adotados previamente (B2). No Projeto Pedagógico deste Curso existe referência clara ao modelo e critérios estabelecidos para essa avaliação. Alguns indicadores são o relacionamento interpessoal, a postura, a iniciativa diante dos problemas, o planejamento das ações, elaboração de relatórios, comunicação verbal e escrita, responsabilidade e ética do aluno, bem como a relação entre teoria e a prática. Os estágios acontecem semanalmente e os estudantes são avaliados ao longo da disciplina promovendo a avaliação formativa. Autore ${ }^{20}$ já mencionaram a importância de diferentes estratégias para promover uma avaliação formativa e técnicas para o feedback ao estudante em cenário clínico.

É responsabilidade do supervisor informar ao aluno sobre o seu desempenho, mas também promover a reflexão e autocrítica. Sugerir mudanças de atitude para atingir o perfil profissional é essen$\mathrm{cial}^{21}$. O fato da maioria dos participantes referir que o supervisor tenha realizado esta função (B6) pode contribuir para o processo de aprendizagem na prática clínica, uma vez que benefícios como o aumento de confiança, da motivação e autoestima do aluno podem ser decorrentes do feedback fornecido pelo supervisor ${ }^{21}$. Uma orientação convergente, com feedback ajustado em tempo e conteúdo, bem como a existência de momentos de reflexão referente às complexas e singulares situações clínicas são fundamentais para os supervisionados obterem melhores resultados. É importante que o supervisor adote uma postura empática e assertiva, criando um clima favorável para a exposição de erros, dificuldades e potencialidades, o que reforça a colaboração mútua e a responsabilidade de todos no desenvolvimento de habilidades técnicas ${ }^{2}$.

Os participantes deste estudo $(77,27 \%)$ concordaram que a observação do atendimento realizado por eles e as anotações (feedback) que o supervisor faz semanalmente no plano diário favorecem o planejamento de ações futuras (B8, 86,36\%). A observação da atuação clínica foi considerada uma atividade positiva, tanto na situação de observador quanto na de observado por supervisionados, com vantagem temporal comparada à prática de transcrição, destacando-se a autorreflexão da prática, o uso de técnicas e a troca de experiência, favorecendo o desenvolvimento de competências profissionais. 
O supervisor deve, portanto, ser um facilitador, mediador do processo construído pelo grupo de supervisão e incentivador do papel do aluno na percepção destes participantes, ajudando a fazer uma leitura compreensiva da realidade, apoiando a inserção na vida profissional e a avaliação das suas capacidades e competências. Deve servir de modelo ao aluno, sendo que a intervenção realizada durante a supervisão deve promover a aprendizagem do supervisionando, por meio da modelagem e do reforço diferencial dos comportamentos adequados do aprendiz.

Desde o primeiro dia de estágio supervisionado, a organização e a clareza das regras da instituição onde estão realizando o atendimento passam a ser um pilar importante para sua formação, assim, é preciso que o estudante sinta-se integrado ao cenário de prática para que possa respeitar as normas de funcionamento de cada unidade de saúde. Assim sendo, a compreensão e a obediência do regulamento dos diferentes cenários de prática $(\mathrm{C} 3)$, também são aspectos relevantes o que foi percebido pela maioria dos participantes $(84,09 \%$ concordaram). Além disso, o estagiário sente a necessidade de desenvolver competências gerais, um saber fazer, saber ser e saber conviver, oportunizadas por estes diferentes cenários de atuação.

Os participantes concordaram que é responsabilidade do estagiário planejar e aplicar as técnicas (C2, 84,09\%) e que devem assumir um papel ativo na construção do conhecimento (C4, 95,45\%), bem como revisar a literatura para sedimentar suas ações $(\mathrm{C} 5,95,45 \%)$, condizente a outros achados, que evidenciaram o processo ensino-aprendizagem contemplando o aluno/supervisionado como agente de seu próprio conhecimento, por meio do processo dialógico com o professor/supervisor e o grupo de supervisão ${ }^{21}$.

\section{Conclusão}

Os estagiários de Fonoaudiologia perceberam a importância da função do supervisor, do papel do estagiário e da supervisão clínica, como modelo de ensino-aprendizagem para a aquisição e aprimoramento de habilidades e competências necessárias para a prática profissional.

Estudos como este subsidiam o melhor entendimento sobre como são realizadas as práticas de supervisão nos cursos de Fonoaudiologia. O relato e discussão sobre diferentes estratégias de aprendizagem adotadas em cenário clínico são fundamentais para melhorar a qualidade de ensino e prática profissional.

\section{Referências}

1. Ribeiro IL, Medeiros Junior A. Graduação em saúde, uma reflexão sobre ensino- aprendizado. Trab educ saúde. 2016; 14 (1): 33-53.

2. Sei MB, Paiva MLSC. Grupo de supervisão em psicologia e a função de holding do supervisor. Psico ensin form. 2011; 2 (1): 9-19.

3. Moreira SBS. Descrição de algumas variáveis em um procedimento de supervisão de terapia analítica do comportamento. Psicol reflex crit. 2003; 16 (1): 157-70.

4. Menezes RLC, Medrado BP. Formação em psicologia clínica: o estágio supervisionado como atividade potencial de desenvolvimento profissional. InterScientia. 2013; 1 (2): 37-51.

5. Alarcão I, Rua M. Interdisciplinaridade, estágios clínicos e desenvolvimento de competências. Texto contexto - enferm. 2005; 14 (3): 373-82.

6. Araújo O, Martins C, Braga F, Macedo AP, Oliveira C, Rosário R. Supervisão em contexto clínico: o testemunho dos estudantes sobre o(s) modelo(s) vigente(s). Ver formac innov educ univers. 2012; 5 (2): 112-21.

7. Teixeira VRV, Souza LAP, Fantini LA, Ferreira LP. Formação do fonoaudiólogo: avaliação discente em supervisão clínica. Distúrb comun. 2009; 21 (3): 327-38.

8. Domingues RCL, Amaral E, Zeferino AMB. Os diferentes olhares na avaliação de alunos em estágio clínico supervisionado. Rev assoc med bras. 2009; 55 (4): 458-62.

9. Barletta JB, Fonseca ALB, Delabrida ZNC. A importância da supervisão de estágio clínico para o desenvolvimento de competências em terapia cognitivo-comportamental. Psicolo teor prát. 2012; 14 (3): 153-67.

10. Lima BPS, Vilela RQB. Características e desafios docentes na supervisão de estágio em fonoaudiologia. Rev CEFAC. 2014; 16 (6): 1962-71.

11. Barreto MC, Barletta JB. A supervisão de estágio em psicologia clínica sob as óticas do supervisor e do supervisionando. Cad graduação - ciênc biol saúde. 2010; 12 (2): $20-30$.

12. Lima TC, Paixão FRC, Cândido EC, Campos CJG, Ceolim MF. Estagio curricular supervisionado: analise da experiência discente. Rev bras enferm. 2014; 67 (1): 133-40.

13. Oliveira TSB, Oliveira e Silva HKS, Barros Filho J, Soares PRD, Souza SO. Possibilidades e limitações: compartilhando experiências vivenciadas no estágio supervisionado em enfermagem. Rev Univers Vale do Rio Verde. 2014; 12 (01): 437-444.

14. Barletta JB, Fonseca ALB, Oliveira MIS. Transcrição e observação como estratégias para aprimoramento da competência clínica. Rev bras terap cogn. 2011; 7 (2): 17-24.

15. Rodrigues R, Mendes MZAD. A formação do supervisor clínico-institucional: a experiência de um percurso. Cad ESP. 2013; 7 (1): 59-67. 
16. Queiroz MAS, Teixeira CLV, Braga CM, Almeida KA, Pessoa RX, Almeida RCA. Estágio curricular supervisionado: percepções do aluno- terapêuta em fonoaudiologia no âmbito hospitalar. Rev CEFAC. 2013. 15 (1): 135-43.

17. Bollela VR, Germani ACCG, Campos HH, Amaral E. Educação baseada na comunidade para as profissões da saúde: aprendendo com a experiência brasileira. Ribeirão Preto, FUNPEC. 2014.

18. Benito GAV, Tristão KM, Paula ACSF, Santos MA, Ataide LJ, Lima RCD. Desenvolvimento de competências gerais durante o estágio supervisionado. Rev bras enferm. 2012; 65 (1): $172-8$.
19. Bitondi FR, Ribeiro AC, Sétem J. O contexto da supervisão grupal: ambiente para formação de terapeutas e de supervisores. Rev bras ter comport cogn. 2012; 14 (1): 31-7.

20. Norcini J, Burch V. Workplace-based assessment as an educational tool: AMEE Guide No. 31. Med Teach 2007; 29 (9): 855-71.

21. Clynes MP, Raftery SEC. Feedback: an essential element of student learning in clinical practice. Nurse educ pract. 2008; 8 (6): 405-11. 